\title{
GenIO: a phenotype-genotype analysis web server for clinical genomics of rare diseases

\author{
Daniel Koile ${ }^{1 \dagger}$, Marta Cordoba ${ }^{2,3+}$, Maximiliano de Sousa Serro ${ }^{1}$, Marcelo Andres Kauffman ${ }^{2,3^{*}}$ \\ and Patricio Yankilevich ${ }^{1 *}$ (D)
}

\begin{abstract}
Background: GenlO is a novel web-server, designed to assist clinical genomics researchers and medical doctors in the diagnostic process of rare genetic diseases. The tool identifies the most probable variants causing a rare disease, using the genomic and clinical information provided by a medical practitioner. Variants identified in a whole-genome, whole-exome or target sequencing studies are annotated, classified and filtered by clinical significance. Candidate genes associated with the patient's symptoms, suspected disease and complementary findings are identified to obtain a small manageable number of the most probable recessive and dominant candidate gene variants associated with the rare disease case. Additionally, following the American College of Medical Genetics and Genomics and the Association of Molecular Pathology (ACMG-AMP) guidelines and recommendations, all potentially pathogenic variants that might be contributing to disease and secondary findings are identified.

Results: A retrospective study was performed on 40 patients with a diagnostic rate of $40 \%$. All the known genes that were previously considered as disease causing were correctly identified in the final inherit model output lists. In previously undiagnosed cases, we had no additional yield.
\end{abstract}

Conclusion: This unique, intuitive and user-friendly tool to assists medical doctors in the clinical genomics diagnostic process is openly available at https://bioinformatics.ibioba-mpsp-conicet.gov.ar/GenlO/.

Keywords: Rare disease, Exome sequencing, Genome sequencing, Clinical informatics, Variant analysis, Bioinformatics

\section{Background}

The advances in genetics, and the growing availability of health and genetic data, are making personal genomics a clinical reality. Clinical implementation of wholegenome sequencing or whole-exome sequencing as a single and primary test, will provide a higher diagnostic yield than conventional testing, while decreasing the number of genetic tests and ultimately the time required to reach a genetic diagnosis [1]. Genetic risk communication and genetic diagnosis will rapidly broadened in scope and practice, as emerging genomic technologies

\footnotetext{
* Correspondence: marcelokauffman@gmail.com; pyankilevich@ibioba-mpspconicet.gov.ar

${ }^{\dagger}$ Equal contributors

${ }^{2}$ Consultorio de Neurogenética, Centro Universitario de Neurología y División Neurología, Hospital J.M. Ramos Mejia, Facultad de Medicina, UBA, Buenos Aires, Argentina

${ }^{1}$ Instituto de Investigación en Biomedicina de Buenos Aires (IBioBA), CONICET

- Partner Institute of the Max Planck Society, Buenos Aires, Argentina

Full list of author information is available at the end of the article
}

allow more medical doctors to access information regarding their patients' genetic makeup [2].

Here we present GenIO, a clinical genomics webtool to assist in the clinical genomics diagnostic process. Through our web server the user uploads the patient's genetic information as a variant call format (VCF) file, and enter the patient's clinical information as structured, comprehensive and well-defined terms for observed symptoms, suspected disease and complementary findings. Starting from thousands of variants, GenIO applies different annotations and filters, in order to identify a small number of the most probable recessive and dominant variants associated with rare Mendelian diseases (Fig. 1).

GenIO clinically classifies all variants using up-to-date clinical information, and identifies those variants with potentially functional pathogenic effects guided by the ClinVar database annotations [3], the Mendelian Clinically Applicable Pathogenicity (M-CAP) classifier [4], and the InterVar clinical interpretation [5] which follows the 


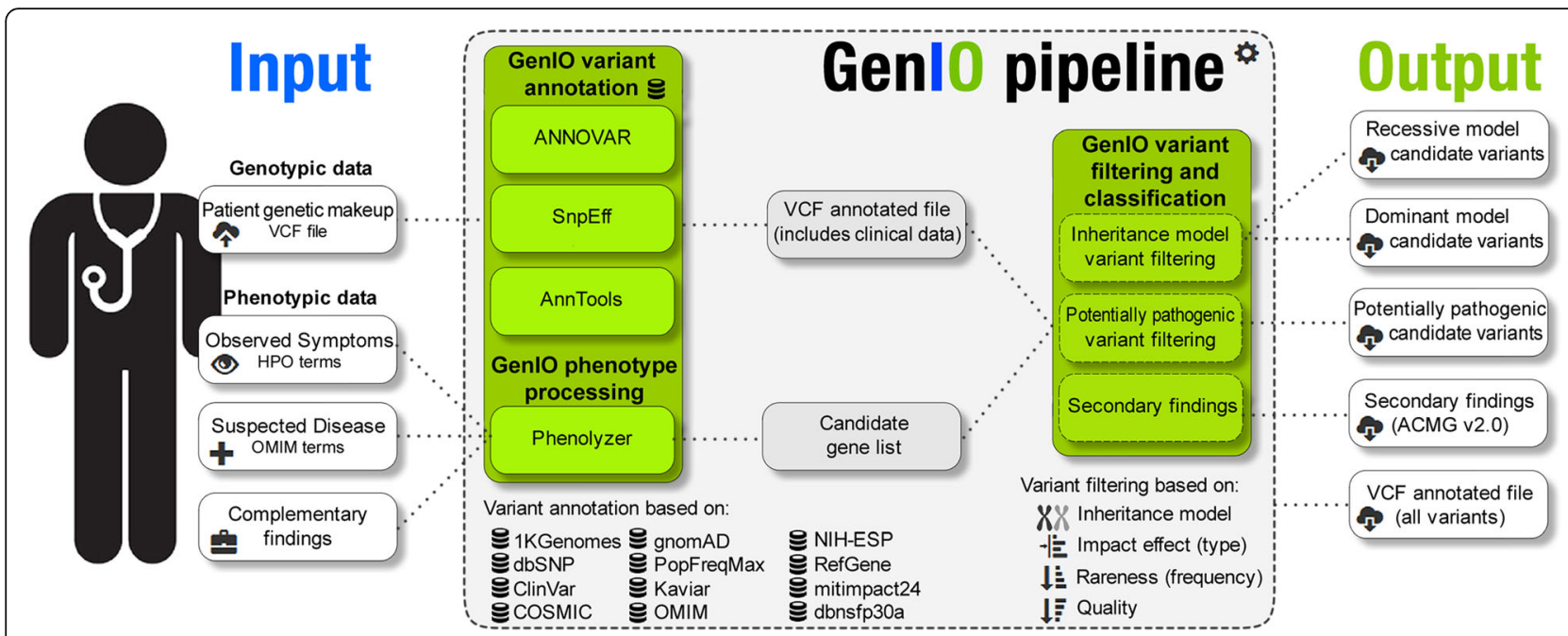

Fig. 1 The GenIO pipeline. From left to right: Input parameters (VCF file and phenotype terms), GenlO pipeline, Output files (candidate recessive and dominant variants lists, potential pathogenic variants list, annotated VCF and secondary findings)

American College of Medical Genetics and Genomics and the Association for Molecular Pathology (ACMGAMP) recommendations [6]. Additionally, GenIO reports secondary findings, in alignment with the ACMG latest recommendations for reporting of secondary findings in clinical exome and genome sequencing [7].

The GenIO process assists the medical practitioner in confirming a diagnosis for the patient case. At the moment, this crucial and time consuming annotation and filtering procedure is being done either manually, by the few geneticists able to benefit from bioinformatical support, or by using more complex web servers such as wAnnovar [8], Omim Explorer [9], eXtasy [10], PhenIX [11] or PhenGen [12], designed for research exploration and not for medical doctors working the diagnosis of rare diseases.

GenIO interface has been designed to minimize usage complexity, allowing medical doctors to input a patient's genetic makeup from a VCF file together with the patient's phenotype, entered as controlled vocabulary terms from the Human Phenotype Ontology (HPO) project [13] and the Online Mendelian Inheritance in Man (OMIM) database (https://omim.org/) in a precise and easy way, to obtain a clear and concise output report (Fig. 2). This simple, intuitive and userfriendly clinical genomics Input-Output process gives GenIO its name.

GenIO is a unique web server, designed for medical doctors and researchers in the field of clinical genomics who may not have the necessary bioinformatics skills to annotate, classify and filter variants identified in highthroughput-sequencing studies to be able to choose the candidate disease causative gene from a small number of the most probable pathogenic variants associated with rare Mendelian disorders.

\section{Implementation}

Benchmark datasets

A benchmark dataset were simulated using a trustable and freely available source of pathogenic variants in the ClinVar public archive, their HPO associated terms, and the exome of a healthy individual to create a set of 125 simulated cases to be tested with GenIO. The ClinVar archive version 20,160,302 was downloaded and processed to filter out non-pathogenic variants, variants with no solid support evidence, and variants that lacked an OMIM registry. The resulting pathogenic gene variants were annotated with their known HPO associated terms downloaded from the Human Phenotype Ontology's project website. Then a publicly available VCF file of the exome of a healthy individual was obtained [14]. Finally, 125 pathogenic gene variants with $\mathrm{HPO}$ annotations were randomly chosen from the filtered and annotated ClinVar file, and each added to different copies of a the exome of a healthy individual, obtaining a dataset of 125 simulated cases (pairs of VCF and HPO terms) to be tested in GenIO. The benchmark dataset is available at https://bioinformatic s.ibioba-mpsp-conicet.gov.ar/GenIO/tests.zip

An additional real dataset of 40 patients from the Neurogenetics Unit in Hospital Ramos Mejía, Buenos Aires, which were previously studied applying WES and Sanger confirmation with a diagnostic rate of $40 \%$ (16 from 40) [15] were used to conduct a retrospective study. The study was approved by the Ethics Committee and Institutional Review Board of our Hospital JM Ramos Mejia, informed written consent was obtained from the participants, and the data were analyzed anonymously.

Finally, to further evaluate and compare the performance of GenIO, a smaller benchmark dataset of 10 cases with definitive diagnosis obtained from the former datasets was 

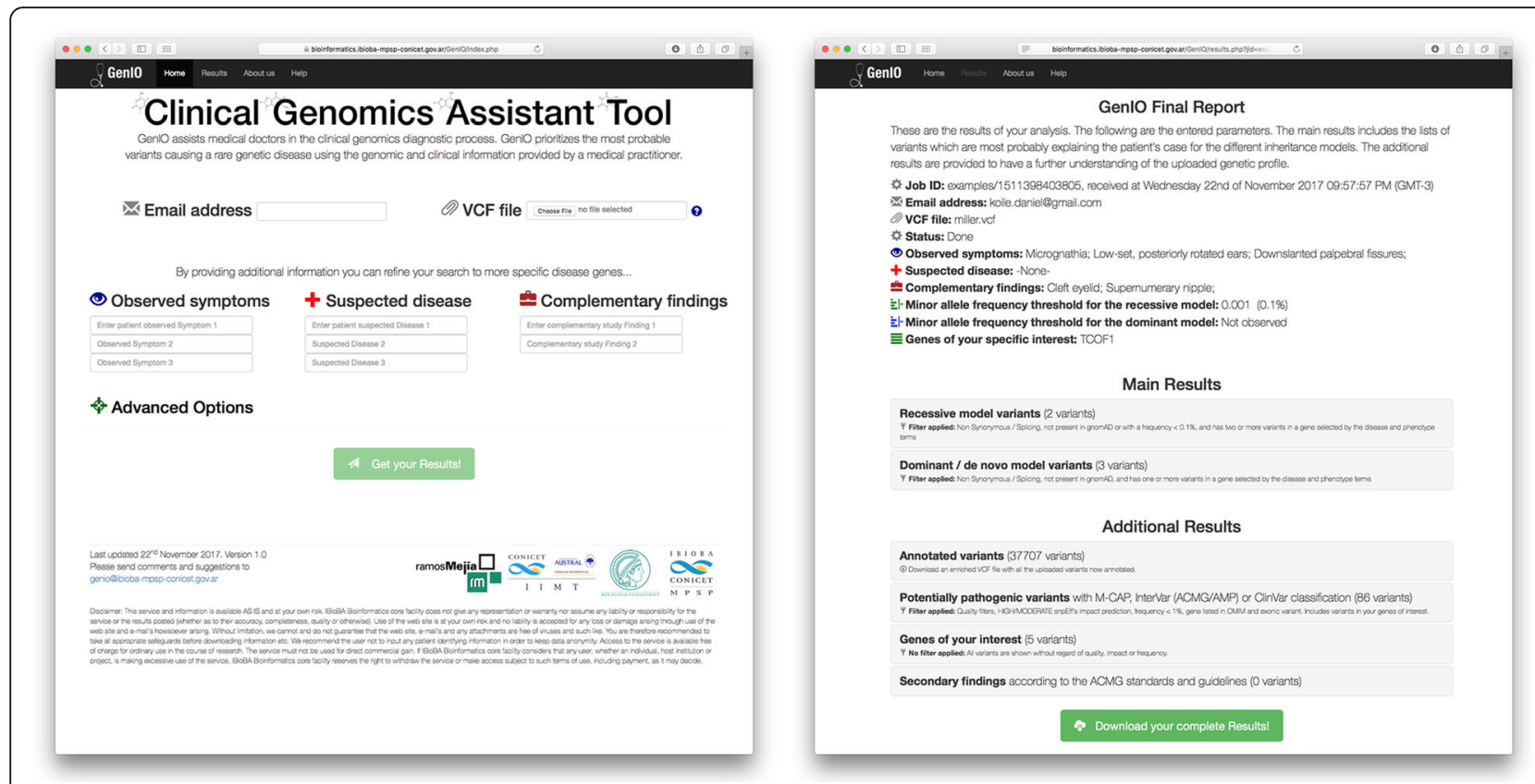

Fig. 2 The GenlO user interface

created to challenge other existing clinical genomics web servers to find the causative gene under the same input parameters.

\section{VCF file validation}

The uploaded VCF file is validated in order to check compliance with the standardized VCF format version 4.0 or higher. The VCF header should contain the format information, and the column names and order as specified by the Global Alliance for Genomics and Health Data Working group file format team (https://samtools.github.io/htsspecs/). VCF columns must be tab separated, have each the proper data type, and have no duplicated variant entries. Only variants that have passed all the quality controls, and hence have a PASS value in the FILTER field, will be taken in consideration for the analysis. In this first release the files uploaded to GenIO must be $200 \mathrm{MB}$ or smaller.

\section{Variant annotation and phenotype processing}

GenIO's variant annotation process uses Annovar [16], Anntools [17], and SnpEff [18] to annotate all variants with information from some of the main clinical genomics databases such as ClinVar, OMIM, the Genome Aggregation Database (gnomAD) [19], and dbSNP [20]; generating a merged and annotated VCF file.

GenIO's phenotype process analyses the symptoms, suspected disease and complementary findings entered terms with Phenolyzer [21] to obtain the list of genes related to the patient's disease/phenotype. Since the candidate genes associated to the entered phenotype are obtained by using Phenolyzer, which has an algorithm to predict putative disease genes, GenIO is then able to identify disease mutations in genes not previously described as being disease-causing.

\section{Variant filtering and classification}

GenIO's variant filtering and classification process identifies the most probable recessive and dominant deleterious variants in the list of genes, related to the patient's disease/phenotype, by filtering on variant effect, population frequency, potential impact, and quality by using the variant_reduction script from Annovar and several custom filters. The default inheritance model output lists include deleterious variants with gnomAD Exome allele frequencies $<0.1 \%$ for the recessive model, and not observed in gnomAD for the dominant model. These variants are then classified by the Mendelian Clinically Applicable Pathogenicity (M-CAP) classifier, the InterVar ACMG-AMP clinical interpretation tool, and the ClinVar clinical significance annotation for the medical doctor to have a better understanding of the candidate causative variants informed. The GenIO's interface advanced options enables the user to enter a specific gene list of interest for analysis, and to modify the filtering thresholds of population frequency according to the rareness of the suspected condition due to default filtering frequencies might be too low for several Mendelian disorders.

An additional list of variants with potentially functional disease-related pathogenic effects is generated by filtering variants in genes involved in Mendelian disorders (present in the OMIM database); with impact on the gene product (nonsense and frameshift mutations, splice site alterations, 
loss of stop codons, non-synonymous substitutions and codon insertions and deletions); with gnomAD Exome allele frequency $<1 \%$; and with a clinical significance of pathogenic or likely-pathogenic nature, obtained either from the ClinVar database, the M-CAP classifier, or the InterVar ACMG-AMP clinical interpretation.

GenIO creates a minimum list of secondary findings, which includes deleterious variants found in 59 medically actionable genes (ACMG SF v2.0), recommended for reporting in clinical genomic sequencing studies.

\section{Server security}

The GenIO application runs on a Secure HTTP Apache web server hosted on our Bioinformatics core facility at the Instituto de Investigación en Biomedicina de Buenos Aires (IBioBA). All GenIO databases and third-party programs used are locally installed on the server, so there is no further information transferred. The user data uploaded in the server is used for GenIO analysis only, stored for one month, and erased afterwards.

\section{Implementation and availability of web server}

The methodology for identifying the most probable variants causing a rare disease described above is implemented in the web server named GenIO, using Linux, Apache, PHP, JavaScript architecture and is made publicly available online at https://bioinformatics.ibiobampsp-conicet.gov.ar/GenIO/

\section{Results}

In order to validate the tool, we conducted a retrospective study on 40 patients with a diagnostic rate of $40 \%$ (16 from 40 cases) from the Neurogenetics Unit in Hospital Ramos Mejía, Buenos Aires. We reanalysed them with GenIO, obtaining, in the final inherit model output lists, all the known genes that were previously considered as disease causing (Additional file 1: Table S1). In previously undiagnosed cases, we had no additional yield. GenIO was also successfully validated with different well known cases such as Miller syndrome in Ng et al., 2010 [22], Nature Genetics (causative gene: $D H O D H$ ), and with Schinzel-

Table 1 Comparison with other web servers features

\begin{tabular}{|c|c|c|c|c|c|c|}
\hline Feature $\backslash$ Web servers & PhenIX & eXtasy & OMIM Explorer & Phen-Gen & wAnnovar & GenlO \\
\hline Year of last update & 2014 & 2013 & 2016 & 2013 & 2015 & 2017 \\
\hline Simple Input interface & Yes & Yes & No & Yes & Yes & Yes \\
\hline Acceptance of VCF files & Yes & Yes & Yes & Yes & Yes & Yes \\
\hline Phenotype (HPO terms) & Yes & Yes & Yes & IDs only & Yes & Yes \\
\hline Phenotype (OMIM terms) & No & No & Yes & No & Yes & Yes \\
\hline List of genes of interest to analyze & No & No & Yes & No & No & Yes \\
\hline Customizable Rareness of the condition & Yes & No & Yes & Yes & Yes & Yes \\
\hline Fast responsive interface & Yes & Yes & No & Yes & Yes & Yes \\
\hline No need of extra files to run & Yes & Yes & Yes & No & Yes & Yes \\
\hline Trio datasets & No & No & No & Yes & No & No \\
\hline Freeform text input & No & No & Yes & No & No & No \\
\hline Email notification & No & Yes & No & Yes & Yes & Yes \\
\hline Dominant and Recessive in single run & Yes & No & Yes & No & Yes & Yes \\
\hline Can be used by a medical practitioner & Yes & No & Yes & No & Yes & Yes \\
\hline Output interface with results & Yes & No & Yes & No & Yes & Yes \\
\hline Simple Output interface & Yes & - & No & - & Yes & Yes \\
\hline ACMG-AMP Classification & No & No & No & No & No & Yes \\
\hline M-CAP Classification & No & No & No & No & No & Yes \\
\hline ClinVar Classification & Top 20 only & No & No & No & Yes & Yes \\
\hline ACMG Secondary findings & No & No & No & No & No & Yes \\
\hline Full VCF annotation & No & No & No & No & Yes & Yes \\
\hline Handful set of candidate variants & Yes & No & Yes & Yes & Yes & Yes \\
\hline Session saving & No & Yes & Yes & Yes & Yes & Yes \\
\hline Phenotype suggestion & No & No & Yes & No & No & No \\
\hline Secure server (https) & No & No & No & No & No & Yes \\
\hline Multi-nucleotide variants & Yes & No & Yes & Yes & Yes & Yes \\
\hline
\end{tabular}


Giedion syndrome in Hoischen et al., 2010 [23], Nature Genetics (causative gene: SETBP1), both included as examples in the GenIO web server.

The benchmarking performed on GenIO with the simulated dataset identified the candidate pathogenic gene variants in the recessive or dominant inheritance models in 94 out of the 125 cases, obtaining a sensitivity of the $75.2 \%$. It should be noted that the inheritance model filters applied in GenIO (see Implementation section) do not rely on the ClinVar clinical significance annotations, making this benchmark completely unbiased. All these tests were run with GenIO default parameters.

We compared GenIO with other existing clinical genomics webtools in terms of features and usability from a clinician user perspective. The compared web servers are wAnnovar, Omim Explorer, eXtasy, PhenIX and Phen-Gen (Table 1).

Finally, to further evaluate the performance of GenIO, we evaluated these same web servers on clinical results comparing 10 of the former analysed cases with definitive diagnosis to find the causative gene under the same input parameters (Additional file 1: Table S2).

\section{Discussion}

GenIO results may enable diagnosis confirmation, and the output information will eventually help to identify the optimal treatment and clinical management for the patient. If, after analysis, the patient still lacks a clear etiology, the output information from GenIO can be used to launch a query on Matchmaker Exchange [24] platform to find additional cases with a deleterious variant in the same listed genes or with overlapping phenotype, which may provide sufficient evidence to identify the causative gene.

The quality of the variants identified in the VCF file uploaded by the user represents limitations to this clinical genomics analysis system. Since the raw sequences or genotype data is pre-processed and filtered before it is saved in a VCF format file, we are not able to ensure the quality of previous data processing, and have to assume an acceptable variant quality, and therefore a trustworthy variant call. We do, nevertheless, validate the format of the VCF file and filter out variants that did not pass the quality thresholds.

Although trio analysis is necessary for the detection of de novo mutations, GenIO does not support this analysis. As the list of de novo variants is usually small enough to be manually interpretable, usually does not require further interpretation.

The manual update of the GenIO's annotation databases represents another limitation to the predictive performance. While clinical research evidence is being generated at ever faster rates, much of this evidence is not readily available in databases. Quality of the databases is also a possible limitation, as clinical databases may include wrong annotations. GenIO works with trustable sources, but nevertheless, they still could contain errors.

\section{Conclusions}

GenIO's intuitive and user-friendly interface was designed to be used not only by clinical genomics researchers, but also by medical doctors. Its simple input interface and the use of controlled vocabulary to enter clinical information minimize spelling and writing errors while entering the patient's phenotypic information. Its diagnosis-oriented output presents only a small manageable number of the most probable recessive and dominant candidate gene variants associated with the rare disease case. Most of the existing clinical genomics web servers supporting diagnosis tasks are scientifically oriented and not designed to be used by medical doctors, on which we experienced some usability problems. In this sense, GenIO is one of the first public web servers developed with the aim of bringing new clinical genomics tools to the medical and scientific community.

Future work will include the identification of pharmacogenomic variants, the development of integrative visualizations for an improvement in the variant clinical interpretation, migration to a cloud computing architecture to handle bigger datasets, the development of a natural language processing of electronic medical records for phenotype suggestions, and the implementation of more ACMG-AMP guidelines and standards.

\section{Availability and requirements}

Project name: GenIO.

Project home page:https://bioinformatics.ibioba-mpspconicet.gov.ar/GenIO/

Operating system(s): Platform independent.

Programming language: Javascript, PHP, GNU-bash shell.

Other requirements: Phenolyzer (v.1.0.5), Annovar (v.2017Jul17)(v.2015Dec14), Anntools (v.1.1), and SnpEff (v.4.2).

License: GNU General Public License.

Any restrictions to use by non-academics: licence needed.

\section{Additional file}

Additional file 1: Table S1. Validated exomes with definitive diagnosis. Table S2. GenlO performance comparison. (DOCX $77 \mathrm{~kb}$ )

\section{Abbreviations}

ACMG SF: ACMG Secondary Findings; ACMG-AMP: American College of Medical Genetics and Genomics and the Association of Molecular Pathology; gnomAD: The Genome Aggregation Database; HPO: Human Phenotype

Ontology; IBioBA: Instituto de Investigación en Biomedicina de Buenos Aires; M-CAP: Mendelian Clinically Applicable Pathogenicity; OMIM: Online Mendelian Inheritance in Man; VCF: Variant Call Format 


\section{Acknowledgements}

All the authors are members of the Argentine National Research Council (CONICET). This work was funded by grants from CONICET, ANPCYT and FOCEM-Mercosur.

\section{Funding}

All the authors are members of the Argentine National Research Council (CONICET). This work was funded by grants from CONICET, ANPCYT and FOCEM-Mercosur.

\section{Availability of data and materials}

Patients data are from the Cordoba M, Rodriguez-Quiroga S, Vega P, Amartino $\mathrm{H}$, Vazquez-Dusefante $\mathrm{C}$, Medina $\mathrm{N}$, et al. Whole Exome Sequencing in Neurogenetic Diagnostic Odysseys: An Argentinian Experience. bioRxiv 060319 (2016) study whose authors may be contacted at the email of the article corresponding author Dr. Marcelo Kauffman (marcelokauffman@gmail.com) to access the anonymized data. The data cannot be publicly deposited due to patient privacy. The benchmarking simulated dataset used and analysed during the system validation are available from the corresponding author on request.

\section{Authors' contributions}

PY MK MC conceived and designed the tool. DK PY MSS implemented the tool. MC MK performed the experiments and comparisons. MC DK MSS MK PY analysed the data. PY MK DK wrote the paper. All authors read and approved the final manuscript.

\section{Ethics approval and consent to participate}

The validation retrospective study was approved by the Ethics Committee and Institutional Review Board of the Hospital JM Ramos Mejia, informed written consent was obtained from all participants, and the data were analysed anonymously.

\section{Consent for publication}

Not applicable.

\section{Competing interests}

The authors declare that they have no competing interests

\section{Publisher's Note}

Springer Nature remains neutral with regard to jurisdictional claims in published maps and institutional affiliations.

\section{Author details}

'Instituto de Investigación en Biomedicina de Buenos Aires (IBioBA), CONICET - Partner Institute of the Max Planck Society, Buenos Aires, Argentina. ${ }^{2}$ Consultorio de Neurogenética, Centro Universitario de Neurología y División Neurología, Hospital J.M. Ramos Mejia, Facultad de Medicina, UBA, Buenos Aires, Argentina. ${ }^{3}$ Programa de Medicina de Precisión y Genómica, Instituto de Investigaciones en Medicina Traslacional, Facultad de Ciencias Biomédicas, Universidad Austral-CONICET, Buenos Aires, Argentina.

\section{Received: 9 October 2017 Accepted: 15 January 2018}

\section{Published online: 27 January 2018}

\section{References}

1. Stavropoulos DJ, et al. Whole-genome sequencing expands diagnostic utility and improves clinical management in paediatric medicine. Genomic Medicine. 2016;1:15012.

2. Lautenbach DM, Christensen KD, Sparks JA, Green RC. Communicating genetic risk information for common disorders in the era of genomic medicine. Annu Rev Genom Human Genetics. 2013;14:491-513.

3. Landrum MJ, Lee JM, Benson M, Brown G, Chao C, Chitipiralla S, et al. ClinVar: public archive of interpretations of clinically relevant variants. Nucleic Acids Res. 2016 Jan:44(D1):D862-8.

4. Jagadeesh KA, Wenger AM, Berger MJ, Guturu H, Stenson PD, Cooper DN, et al. M-CAP eliminates a majority of variants of uncertain significance in clinical exomes at high sensitivity. Nat Genet. 2016 Oct;48(12):1581-6.

5. Li Q, Wang K. Intervar: clinical interpretation of genetic variants by the 2015 ACMG-AMP guidelines. Am J Hum Genet. 2017;100(2):267-280.

6. Richards S, Aziz N, Bale S, Bick D, Das S, Gastier-Foster J, et al. Standards and guidelines for the interpretation of sequence variants: a joint consensus recommendation of the American College of Medical Genetics and Genomics and the Association for Molecular Pathology. Genetics in Medicine. 2015 May; 17(5):405-24.

7. Kalia SS, Adelman K, Bale SJ, Chung WK, Eng C, Evans JP, et al. Recommendations for reporting of secondary findings in clinical exome and genome sequencing, 2016 update (ACMG SF v2.0): a policy statement of the American College of Medical Genetics and Genomics. Genetics in Medicine. 2016 Nov;19(2):249-55.

8. Yang $\mathrm{H}$, Wang K. Genomic variant annotation and prioritization with ANNOVAR and wANNOVAR. Nat Protoc. 2015 Sep;10(10):1556-66.

9. James RA, Campbell IM, Chen ES, Boone PM, Rao MA, Bainbridge MN, et al. A visual and curatorial approach to clinical variant prioritization and disease gene discovery in genome-wide diagnostics. Genome Med. 2016;8(1):13.

10. Sifrim A, Popovic D, Tranchevent LC, Ardeshirdavani A, Sakai R, Konings P, et al. eXtasy: variant prioritization by genomic data fusion. Nat Methods. 2013 Nov;10(11):1083-4

11. Zemojtel T, Köhler S, Mackenroth $L$, Jäger $M$, Hecht J, Krawitz $P$, et al. Effective diagnosis of genetic disease by computational phenotype analysis of the disease-associated genome. Sci Transl Med. 2014;6(252):252ra123.

12. Javed A, Agrawal S, Ng PC. Phen-gen: combining phenotype and genotype to analyze rare disorders. Nat Methods. 2014 Sep;11(9):935-7.

13. Köhler S, Vasilevsky NA, Engelstad M, Foster E, McMurry J, Aymé S, et al. The human phenotype ontology in 2017. Nucleic Acids Res. 2017 Jan:45(D1):D865-76.

14. Glusman G, Cariaso M, Jimenez R, Swan D, Greshake B, Bhak J, et al. Low budget analysis of direct-to-consumer genomic testing familial data. F1000Research. 2012;1:3.

15. Cordoba M, Rodriguez-Quiroga S, Vega P, Amartino H, Vazquez-Dusefante C, Medina $\mathrm{N}$, et al. Whole Exome Sequencing in Neurogenetic Diagnostic Odysseys: An Argentinian Experience. bioRxiv 060319. 2016. https://doi.org/ 10.1101/060319

16. Wang K, Li M, Hakonarson H. ANNOVAR: functional annotation of genetic variants from high-throughput sequencing data. Nucleic Acids Res. 2010 Sep;38(16):e164

17. Makarov V, O'Grady T, Cai G, Lihm J, Buxbaum JD, Yoon S. AnnTools: A comprehensive and versatile annotation toolkit for genomic variants. Bioinformatics (Oxford, England). 2012 Mar;28(5):724-5.

18. Cingolani P, Platts $A$, Wang le $L$, Coon $M$, Nguyen $T$, Wang $L$, et al. A program for annotating and predicting the effects of single nucleotide polymorphisms, SnpEff: SNPs in the genome of Drosophila Melanogaster strain w1118; iso-2; iso-3. Fly. 2012 Apr;6(2):80-92.

19. Lek M, Karczewski KJ, Minikel EV, Samocha KE, Banks E, Fennell T, et al. Analysis of protein-coding genetic variation in 60,706 humans. Nature. 2016 Aug;536(7616):285-91.

20. Sherry ST, Ward MH, Kholodov M, Baker J, Phan L, Smigielski EM, et al. dbSNP: the NCBI database of genetic variation. Nucleic Acids Res. 2001 Jan;29(1):308-11.

21. Yang $\mathrm{H}$, Robinson PN, Wang K. Phenolyzer: phenotype-based prioritization of candidate genes for human diseases. Nat Methods. 2015 Sep;12(9):841-3.

22. Ng SB, Buckingham KJ, Lee C, Bigham AW, Tabor HK, Dent KM, et al. Exome sequencing identifies the cause of a mendelian disorder. Nat Genet. 2009 Nov:42(1):30-5.

23. Hoischen A, van Bon BWM, Gilissen C, Arts P, van Lier B, Steehouwer M, et al. De novo mutations of SETBP1 cause Schinzel-Giedion syndrome. Nat Genet. 2010 Jun;42(6):483-5

24. Philippakis AA, Azzariti DR, Beltran S, Brookes AJ, Brownstein CA, Brudno M, et al. The matchmaker exchange: a platform for rare disease gene discovery Hum Mutat. 2015 Oct:36(10):915-21.

\section{Submit your next manuscript to BioMed Central and we will help you at every step:}

- We accept pre-submission inquiries

- Our selector tool helps you to find the most relevant journal

- We provide round the clock customer support

- Convenient online submission

- Thorough peer review

- Inclusion in PubMed and all major indexing services

- Maximum visibility for your research

Submit your manuscript at www.biomedcentral.com/submit
Biomed Central 